\title{
Article
}

\section{Redressing the balance: Commentary on "Examining motor learning in older adults using analogy instruction" by Tse, Wong, and Masters (2017)}

Bobrownicki, R., Collins, D., Sproule, J., and MacPherson, A.

Available at http://clok.uclan.ac.uk/23424/

Bobrownicki, R., Collins, D. ORCID: 0000-0002-7601-0454, Sproule, J., and MacPherson, A. (2018) Redressing the balance: Commentary on "Examining motor learning in older adults using analogy instruction" by Tse, Wong, and Masters (2017). Psychology of Sport and Exercise, 38 . pp. 211-214. ISSN 1469-0292

It is advisable to refer to the publisher's version if you intend to cite from the work. http://dx.doi.org/10.1016/j.psychsport.2018.05.014

For more information about UCLan's research in this area go to http://www.uclan.ac.uk/researchgroups/ and search for <name of research Group>.

For information about Research generally at UCLan please go to http://www.uclan.ac.uk/research/

All outputs in CLoK are protected by Intellectual Property Rights law, including Copyright law. Copyright, IPR and Moral Rights for the works on this site are retained by the individual authors and/or other copyright owners. Terms and conditions for use of this material are defined in the policies page. 
1 Running head: Commentary on Tse, Wong, and Masters (2017)

2

3

4

5

6

7

8

9 Redressing the balance: Commentary on "Examining motor learning in older adults using analogy

10 instruction" by Tse, Wong, and Masters (2017)

11

12

13

14

15

16

17

18

19

20 

instruction may help older adults acquire resilient motor skills that require reduced cognitive

4 processing compared to traditional explicit instruction. Although we do not dispute that analogy

5 learning may prove useful for this population, in this commentary, we contend that there are

6 methodological issues in this research — which are shared with previous studies comparing analogy

7 and explicit instruction - that potentially limit ecological validity, impact the size of detected effects,

8 influence the development and understanding of associated theory, and, as such, constrain resulting

9 recommendations for applied practice. Of particular concern is the comparison of the single-item

10 analogy instruction to the list of nine explicit instructions, which risks conflating the effects of the

11 type of instruction with the volume of instruction. We further argue that the benefits of analogy may

12 be more parsimoniously explained by the instruction's capability to succinctly convey skill (rather

13 than its potential for limiting reinvestment), but that this capability may only be realised if the to-be-

14 learned analogy is relevant and readily understood by the learner. Finally, we suggest that research in

15 this area must look to incorporate more rigorous methods that compare experimental conditions to

16 representative reference groups that allow us to explore how and when to deploy the myriad

17 instructional tools available to practitioners and learners.

24 Keywords: motor learning, instruction, explicit instruction, analogy, coaching 
In a recent study, Tse, Wong, and Masters (2017) investigated the efficacy of analogy

2 instruction in motor learning for older adults. The authors suggested that older adults may benefit

3 from analogy instructions, as the analogy learners demonstrated more robust performance under

4 pressure and reported fewer verbal rules than their explicit-learning counterparts. These findings

5 clearly correspond with previous research with young adults (e.g., Lam, Maxwell, \& Masters, 2009a,

6 2009b; Liao \& Masters, 2001) and, more recently, with adolescents (Tse, Fong, Wong, \& Masters,

7 2017). We are concerned, however, that limitations in the study by Tse, Wong, et al. (2017), which

8 notably reflect consistent and prevalent issues in the associated literature, continue to skew the debate

9 concerning analogies and explicit instructions, making it more difficult for applied practitioners to

10 equitably evaluate the available instructional tools. This commentary sets forth these issues and

11 presents suggestions for future research in this area.

1. Quantity and quality of instructions may confound the control condition and misrepresent practice

As shown in table 1, explicit instructions have traditionally outnumbered analogy instructions throughout the literature by margins that misrepresent real-world settings (Bobrownicki, MacPherson, Coleman, Collins, \& Sproule, 2015). In their recent study, Tse, Wong, et al. (2017) have continued to follow this customary, but arguably unrepresentative, research paradigm by comparing a single analogy to nine explicit instructions. According to Lam et al. (2009b), in studies such as this, a "fairer comparison might be achieved" (p. 189) by matching the number of rules for these instruction types. Indeed, Bobrownicki et al. (2015) stressed that research in analogy and explicit instruction should aim to avoid these disparate instructional protocols, because these informational imbalances create questionable reference groups, present issues with working memory capacity, and conflict with recommended coaching practice (e.g., Mannie, 1998; McQuade, 2003; Schmidt \& Wrisberg, 2004; UK Athletics, 2009), serving to limit the relevance and generalisability of any findings.

24 Unfortunately, only a few studies to this point have looked to implement such controls on instructional quantity (Bobrownicki et al., 2015; Schücker, Ebbing, \& Hagemann, 2010; Tse, Fong, et al., 2017). Although Tse, Wong, et al. (2017) acknowledged this issue concerning the number of

27 instructions toward the end of the discussion section, the instructional imbalance required greater 
1 attention given the previous criticisms, the limited ecological validity, and the implications for applied

2 practice. As many of the same authors (Tse, Fong, et al., 2017) have previously acknowledged that

3 real-world instruction is ordinarily provided in a step-by-step manner (i.e., one or two instructions at a

4 time) rather than many instructions all at once, it is not necessarily clear why this apparent imbalance

5 continues to persist in the analogy and explicit instruction literature.

6 In addition to issues of quantity, however, there are also concerns regarding the quality of the 7 explicit instructions in the study of Tse, Wong, et al. (2017), as explicit learners were provided

8 specific movement information that possessed limited correspondence to the analogy instruction and 9 the aims of the task. For example, the explicit rules to position feet 'side on at $45^{\circ}$ to the table' and to 10 rotate 'hips, waist, and shoulders forward when serving' provided excess information that was neither 11 conveyed in the single analogy instruction to "move the racket such that it is travelling up the side of a mountain' nor pertinent to the top-spin forehand return task, which did not include any service (balls were delivered to participants in the return task by machine). As research has indicated that analogies may be differentially effective and interact with characteristics of the learner, such as culture (Poolton, Masters, \& Maxwell, 2003) or skill level (Schlapkohl, Hohmann, \& Raab, 2012), quality and relevance of these instruction types deserve much needed attention from researchers and 17 practitioners alike.

\section{Selection of control conditions may overstate or mask effects}

According to Goginsky and Collins (1996), the selection and design of the control or reference 21 groups represent critical considerations for sport psychology researchers, as it has been empirically demonstrated that unrealistic control groups can influence effect sizes and confound results compared to more representative controls (e.g., Winter \& Collins, 2013). With this in mind, the long lists of explicit instructions found in the study of Tse, Wong, et al. (2017) — and many of those studies in

25 Table 1-may not only limit ecological validity, but the resulting, imbalanced experimental comparisons may also empirically overstate the benefits of analogy learning. 
For instance, this issue was highlighted in the study of Bobrownicki et al. (2015) where the

2 effect sizes, when compared to the analogy condition in a jumping task, were $d=1.44$ for the

3 "traditional explicit" condition (eight unique explicit rules to reflect traditional explicit conditions),

4 but $d=.83$ for the "explicit light" condition (number of words reduced to match to analogy

5 instructions). Although the analogy learners still demonstrated the most efficient jumping technique on average compared to the explicit light and traditional explicit conditions, respectively, these differences were not statistically significant and, crucially, the reduction in instructional volume appeared to mitigate the deleterious effects typically associated with explicit instruction. These results suggest that the research of Tse, Wong, et al. (2017)—and many of those from Table 1 —could be overestimating analogy's benefits by conflating the effects of the type of instruction with the volume

11 of instruction through unrepresentative reference groups. Of course, this interpretation does not preclude that analogies are offering genuine - if perhaps smaller than originally stated—advantages to learners over explicit methods (e.g., integration of movement subcomponents, Gestalt processing, differential working memory consumption), but that these findings are being confounded or masked by pervasive instructional inequalities. researchers to corroborate these hypotheses or further explore these concepts. Tieleman (2008) conducted pilot studies examining the quality and quantity of rule-based instruction, which would later be used to inform the work of Schlapkohl, Hohmann, and Raab (2012), but the original data remain unpublished. Tse, Fong, et al. (2017) and Schücker et al. (2010) also matched the number of

21 instructions for the analogy and explicit instruction groups, but both analogy and explicit conditions in these studies were provided with quantities of instructions (11 rules and 30 rules, respectively) that may have exceeded participants' working memory capacities, as suggested by Ille and Cadopi (1999).

24 The issues of working memory capacity notwithstanding, these investigations produced mixed results with Tse, Fong, et al. (2017) observing that adolescent analogy learners performed more robustly under a secondary task load, while Schücker et al. (2010) did not find a benefit for analogy learning under pressure conditions compared to traditional explicit methods. It is conceivable that these 
1 aforementioned issues with instructional quality, quantity, and selection of reference groups have

2 contributed to the "somewhat inconsistent" (p. 15) results for analogy instruction, as identified by

3 Gröpel and Mesagno (2017) in a recent review of choking interventions.

4 3. The value of analogy may lie in its concision

5 Analogy has been presented as a means of forestalling skill failure under pressure (Lam et al.,

6 2009a); however, the premise that analogy instruction engenders less verbal knowledge and, in turn,

7 limits potential for reinvestment is largely predicated, to date, on research that succumbs to issues

8 highlighted under the first two subheadings. Indeed, it should not be surprising to Tse, Wong, et al.

9 (2017) that participants asked to memorise nine instructions (Table 1) might perform less robustly

10 under pressure and report more verbal rules at the conclusion of the study than in a single-instruction

11 analogy condition. As Bobrownicki et al. (2015) argued, analogy's greatest strength, at least from an

12 applied perspective, may instead rest on its potential to parsimoniously deliver relevant instructions,

13 succinctly compiling information regarding movement subcomponents into an easy-to-deliver

14 package. Harking back to earlier, more cognitive considerations of effective coaching, analogies can

15 provide opportunities to make connections to previously embedded concepts that might otherwise be

16 delivered in several explicit chunks. They are also noticeably simpler, helping to conserve important

17 capacity in short-term working memory (cf. Cowan, 2001).

4. Establishing relevance and understanding

The potential for an analogy to deliver on its abbreviated form can only be realised, however, if

20 the to-be-instructed analogy is relevant and readily understood by learners. In this regard, previous

21 findings have suggested that a range of factors, such as cultural and individual differences, can affect analogy effectiveness (Poolton, Masters, \& Maxwell, 2007). For example, the same table tennis analogy (pretend to draw a right-angled triangle with the bat) that was previously successful with

24 English speakers (Liao \& Masters, 2001) proved ineffective for Chinese-speaking participants

25 (Poolton et al., 2003). Of course, such issues should also affect explicit instructions too, as the use and 26 understanding of language is thought to vary from person to person (Reed, 1996). Despite this,

27 however, Tse, Wong, et al. (2017), like most studies before (see Table 1), have not incorporated any 
1 checks to probe participant understanding of either type of verbal instruction. We would posit that

2 participant understanding may represent an even more critical consideration than loyalty to any

3 specific type of instruction which warrants careful emphasis from researchers and practitioners alike

4 as they develop research and practice in the future.

\section{Future directions}

While Bobrownicki et al. (2015) did aim to control instruction quantity, the next steps might involve systematically controlling both the quantity and quality of the analogy and explicit instructions. This would enable the exploration of effective and ineffective analogy and explicit instructions (e.g., complexity; valence; familiarity; learning versus performance; short-term versus long-term effects; and interactions with the needs, preferences, and characteristics of learners and performers). At present, there exists limited scientifically informed guidance for either the development of effective verbal instructions or the pre-emption of potential issues, so this proposed

13 line of enquiry represents necessary research for informing applied practice, especially as earlier 14 investigations have indicated that analogies may not be universally effective (e.g., English- versus 15 Chinese-speaking participants; Poolton, Masters, \& Maxwell, 2003). Given the inconsistent findings to date (Gröpel \& Mesagno, 2017), the relatively small sample sizes common throughout the literature will require attention, as adequately powered studies represent prerequisites for systematic replications and for controlling rates of error (Schweizer \& Furley, 2016). It may be that the above recommendations constitute a research programme that is achievable through Mellers, Hertwig, and

20 Kahneman's (2001) guidelines for adversarial collaboration, which call for joint research under mutually agreed protocols to address opposing interpretations or experimental refutations (we should stress that adversarial is the adjective chosen by Mellers et al. and not one that we would otherwise use to describe Tse, Wong, et al.).

While quality and quantity may represent primary concerns at present, we do not rule out that

25 the effects of these verbal instruction types are moderated only by these. For instance, given the 26 literature's predicted, distinct pathways for implicit and explicit approaches (e.g., Zhu, Poolton, \& 27 Masters, 2012), it may or may not be that analogy and explicit instructions differentially activate 
1 specific areas or processes of the brain. From our perspective, however, it is first necessary to address

2 the concerns regarding quantity and quality before these other possible moderators, such as cortical

3 activation or multimodal learning, can be appropriately investigated.

\section{$4 \quad$ 6. Concluding thoughts}

5 Through the course of this commentary, we have raised issues regarding the study of Tse,

6 Wong, et al. (2017), many of which also pervade the literature as cited in Table 1. In doing this, we

7 are not disputing that analogies represent a potentially useful tool for practitioners; indeed, the

8 rationale is sound and makes intuitive sense, especially if mechanistic explanations consider wider

9 and perhaps more parsimonious explanations than a sole reliance on reinvestment. Rather, we are

10 concerned that methodological shortcomings may be misrepresenting or overstating their usefulness,

11 while also holding back research and practice. For us, given the complexity of real-world sporting

12 environments, analogy represents one of a number of useful tools (e.g., constraints, external focus of

13 attention, demonstration, understanding) that practitioners may dynamically adopt to suit the athlete,

14 the sport, the learning/performance environment, and the desired outcomes (e.g., short-term

15 adjustment versus long-term development). None of these available instructional tools is universally

16 effective, however, and application cannot occur directly from theory without consideration of both

17 their strengths and limitations (Abraham \& Collins, 2011). Going forward, rather than rely on

18 mismatched and artificial comparison groups to promote the efficacy of analogies, research should

19 aim to compare them to representative reference groups, while also systematically investigating how,

20 when, and why they might work. 
2 Abraham, A., \& Collins, D. (2011). Taking the next step: Ways forward for coaching science. Quest, $63,366-384$.

Bobrownicki, R., MacPherson, A. C., Coleman, S. G. S., Collins, D., \& Sproule, J. (2015). Reexamining the effects of verbal instructional type on early stage motor learning. Human Movement Science, 44, 168-181.

Cowan, N. (2001). The magical number 4 in short-term memory: A reconsideration of mental storage capacity. Behavioral and Brain Sciences, 24, 87-114.

9 Goginsky, A. M., \& Collins, D. (1996). Research design and mental practice. Journal of Sports Sciences, 14, 381-392.

Gröpel, P., \& Mesagno, C. (2017). Choking interventions in sports: A systematic review. International Review of Sport and Exercise Psychology, 1-26.

Hu, G., \& Xu, B. (2009). An experimental study on the performance of motor skills acquired implicitly under pressure. China Sport Science, 29, 57-61.

Ille, A., \& Cadopi, M. (1999). Memory for movement sequences in gymnastics: Effects of age and skill level. Journal of Motor Behavior, 31, 290-300.

Koedijker, J. M., Oudejans, R. R. D., \& Beek, P. J. (2007). Explicit rules and direction of attention in learning and performing the table tennis forehand. International Journal of Sport Psychology,

Koedijker, J. M., Poolton, J. M., Maxwell, J. P., Oudejans, R. R. D., Beek, P. J., \& Masters, R. S. W. (2011). Attention and time constraints in perceptual-motor learning and performance: Instruction, analogy, and skill level. Consciousness and Cognition, 20, 245-256. motor skills under pressure. Journal of Sport \& Exercise Psychology, 31, 337-357. 
1 Lam, W. K., Maxwell, J. P., \& Masters, R. S. W. (2009b). Analogy versus explicit learning of a

Law, J., Masters, R. S. W., Bray, S. R., Eves, F. F., \& Bardswell, I. (2003). Motor performance as a function of audience affability and metaknowledge. Journal of Sport \& Exercise Psychology, 25, 484-500.

Liao, C. M., \& Masters, R. S. W. (2001). Analogy learning: A means to implicit motor learning. Journal of Sports Sciences, 19, 307-319.

Mannie, K. (1998). Coaching Through Demonstration. Coach and Athletic Director, 68, 74-75.

McQuade, S. (2003). How to coach sports effectively. Leeds: Coachwise Solutions.

Mellers, B., Hertwig, R., \& Kahneman, D. (2001). Do frequency representations eliminate conjunction effects? An exercise in adversarial collaboration. Psychological Science, 12, 269275.

Poolton, J. M., Masters, R. S. W., \& Maxwell, J. P. (2003). Analogy learning as a chunking mechanism. Hong Kong: Paper presented at the Hong Kong Student Conference in Sport Medicine, Rehabilitation, and Exercise Science.

Poolton, J. M., Masters, R. S. W., \& Maxwell, J. P. (2006). The influence of analogy learning on decision-making in table tennis: Evidence from behavioural data. Psychology of Sport and Exercise, 7, 677-688.

Poolton, J. M., Masters, R. S. W., \& Maxwell, J. P. (2007). The development of a culturally appropriate analogy for implicit motor learning in a Chinese population. The Sport Psychologist, $21,375-382$.

Reed, E. S. (1996). Encountering the world: Toward an ecological psychology. New York: Oxford University Press. 
1 Schlapkohl, N., Hohmann, T., \& Raab, M. (2012). Effects of instructions on performance outcome and movement patterns for novices and experts in table tennis. International Journal of Sport Psychology, 43, 522-541.

Schmidt, R. A., \& Wrisberg, C. A. (2004). Motor learning and performance (3rd ed.). Champaign, Il: Human Kinetics.

Schücker, L., Ebbing, L., \& Hagemann, N. (2010). Learning by analogies: Implications for performance and attentional processes under pressure. Human Movement, 11, 191-199.

Schücker, L., Hagemann, N., \& Strauss, B. (2013). Analogy vs. technical learning in a golf putting task: An analysis of performance outcomes and attentional processes under pressure. Human Movement, 14, 175-184.

Schweizer, G., \& Furley, P. (2016). Reproducible research in sport and exercise psychology: The role of sample sizes. Psychology of Sport and Exercise, 23, 114-122.

Tielemann, N. (2008). Modifikation motorischer Lernprozesse durch Instruktionen. Wirksamkeit von Analogien und Bewegungsregeln. Leipzig, Germany.

Tse, A. C. Y., Fong, S. S. M., Wong, T. W. L., \& Masters, R. S. W. (2017). Analogy motor learning by young children: A study of rope skipping. European Journal of Sport Science, 17, 152-159.

Tse, A. C. Y., Wong, T. W. L., \& Masters, R. S. W. (2017). Examining motor learning in older adults using analogy instruction. Psychology of Sport and Exercise, 28, 78-84.

UK Athletics. (2009). UKCC level 1: Assistant coach coaching manual.

Winter, S., \& Collins, D. (2013). Does priming really put the gloss on performance? Journal of Sport \& Exercise Psychology, 35, 299-307.

Zhu, F., Poolton, J. M., \& Masters, R. S. W. (2012). Neuroscientific aspects of implicit motor learning in sport. In A. Gollhofer, W. Taube, \& J. B. Nielson (Eds.), Routledge handbook of motor control and motor learning (1st ed., p. 432). Abingdon, UK: Routledge. 